\title{
A Strong Convergence Theorem for a Family of Quasi- $\phi$-Nonexpansive Mappings in a Banach Space
}

\author{
Haiyun Zhou' ${ }^{1}$ and Xinghui Gao ${ }^{2}$ \\ ${ }^{1}$ Department of Mathematics, Shijiazhuang Mechanical Engineering College, Shijiazhuang 050003, China \\ ${ }^{2}$ College of Mathematics and Computer Science, Yanan University, Yanan 716000, China
}

Correspondence should be addressed to Haiyun Zhou, witman66@yahoo.com.cn

Received 26 May 2009; Revised 29 August 2009; Accepted 14 September 2009

Recommended by Nan-jing Huang

The purpose of this paper is to propose a modified hybrid projection algorithm and prove a strong convergence theorem for a family of quasi- $\phi$-nonexpansive mappings. The strong convergence theorem is proven in the more general reflexive, strictly convex, and smooth Banach spaces with the property $(\mathrm{K})$. The results of this paper improve and extend the results of S. Matsushita and W. Takahashi (2005), X. L. Qin and Y. F. Su (2007), and others.

Copyright (C) 2009 H. Zhou and X. Gao. This is an open access article distributed under the Creative Commons Attribution License, which permits unrestricted use, distribution, and reproduction in any medium, provided the original work is properly cited.

\section{Introduction}

It is well known that, in an infinite-dimensional Hilbert space, the normal Mann's iterative algorithm has only weak convergence, in general, even for nonexpansive mappings. Consequently, in order to obtain strong convergence, one has to modify the normal Mann's iteration algorithm, the so-called hybrid projection iteration method is such a modification.

The hybrid projection iteration algorithm (HPIA) was introduced initially by Haugazeau [1] in 1968. For 40 years, HPIA has received rapid developments. For details, the readers are referred to papers [2-7] and the references therein.

In 2005, Matsushita and Takahashi [5] proposed the following hybrid iteration method with generalized projection for relatively nonexpansive mapping $T$ in a Banach space $E$ :

$$
\begin{aligned}
& x_{0} \in C \text { chosen arbitrarily, } \\
& y_{n}=J^{-1}\left(\alpha_{n} J x_{n}+\left(1-\alpha_{n}\right) J T x_{n}\right), \\
& C_{n}=\left\{z \in C: \phi\left(z, y_{n}\right) \leq \phi\left(z, x_{n}\right)\right\}, \\
& Q_{n}=\left\{z \in C:\left\langle x_{n}-z, J x_{0}-J x_{n}\right\rangle \geq 0\right\}, \\
& x_{n+1}=\prod_{C_{n} \cap Q_{n}}\left(x_{0}\right) .
\end{aligned}
$$


They proved the following convergence theorem.

Theorem MT. Let E be a uniformly convex and uniformly smooth Banach space, let $C$ be a nonempty closed convex subset of $E$, let $T$ be a relatively nonexpansive mapping from $C$ into itself, and let $\left\{\alpha_{n}\right\}$ be a sequence of real numbers such that $0 \leq \alpha_{n}<1$ and $\limsup _{n \rightarrow \infty} \alpha_{n}<1$. Suppose that $\left\{x_{n}\right\}$ is given by (1.1), where $J$ is the normalized duality mapping on $E$. If $F(T)$ is nonempty, then $\left\{x_{n}\right\}$ converges strongly to $\Pi_{F(T)} x_{0}$, where $\Pi_{F(T)}(\cdot)$ is the generalized projection from $C$ onto $F(T)$.

In 2007, Qin and Su [2] proposed the following hybrid iteration method with generalized projection for relatively nonexpansive mapping $T$ in a Banach space $E$ :

$$
\begin{aligned}
& x_{0} \in C \text { chosen arbitrarily, } \\
& z_{n}=J^{-1}\left(\beta_{n} J x_{n}+\left(1-\beta_{n}\right) J T x_{n}\right), \\
& y_{n}=J^{-1}\left(\alpha_{n} J x_{n}+\left(1-\alpha_{n}\right) J T z_{n}\right), \\
& C_{n}=\left\{v \in C: \phi\left(v, y_{n}\right) \leq \alpha_{n} \phi\left(v, x_{n}\right)+\left(1-\alpha_{n}\right) \phi\left(v, z_{n}\right)\right\}, \\
& Q_{n}=\left\{v \in C:\left\langle x_{n}-v, J x_{0}-J x_{n}\right\rangle \geq 0\right\}, \\
& x_{n+1}=\prod_{C_{n} \cap Q_{n}}\left(x_{0}\right) .
\end{aligned}
$$

They proved the following convergence theorem.

Theorem QS. Let E be a uniformly convex and uniformly smooth Banach space, let $C$ be a nonempty closed convex subset of $E$, let $T$ be a relatively nonexpansive mapping from $C$ into itself such that $\operatorname{Fix}(T) \neq \emptyset$. Assume that $\left\{\alpha_{n}\right\}$ and $\left\{\beta_{n}\right\}$ are sequences in $[0,1]$ such that $\lim _{\sup } \operatorname{sum}_{n \rightarrow \infty} \alpha_{n}<1$ and $\beta_{n} \rightarrow 1$. Suppose that $\left\{x_{n}\right\}$ is given by (1.2). If $T$ is uniformly continuous, then $\left\{x_{n}\right\}$ converges strongly to $\Pi_{\mathrm{Fix}(T)} x_{0}$.

Question 1. Can both Theorems MT and QS be extended to more general reflexive, strictly convex, and smooth Banach spaces with the property $(\mathrm{K})$ ?

Question 2. Can both Theorems MT and QS be extended to more general class of quasi- $\phi$ nonexpansive mappings?

The purpose of this paper is to give some affirmative answers to the questions mentioned previously, by introducing a modified hybrid projection iteration algorithm and by proving a strong convergence theorem for a family of closed and quasi- $\phi$-nonexpansive mappings by using new analysis techniques in the setting of reflexive, strictly convex, and smooth Banach spaces with the property $(\mathrm{K})$. The results of this paper improve and extend the results of Matsushita and Takahashi [5], Qin and Su [2], and others.

\section{Preliminaries}

In this paper, we denote by $X$ and $X^{*}$ a Banach space and the dual space of $X$, respectively. Let $C$ be a nonempty closed convex subset of $X$. We denote by $J$ the normalized duality mapping 
from $X$ to $2^{X^{*}}$ defined by

$$
J(x)=\left\{j \in X^{*}:\langle x, j\rangle=\|x\|^{2}=\|j\|^{2}\right\},
$$

where $\langle\cdot, \cdot\rangle$ denotes the generalized duality pairing between $X$ and $X^{*}$. It is well known that if $X$ is reflexive, strictly convex, and smooth, then $J: X \rightarrow X^{*}$ is single-valued, demicontinuous and strictly monotone (see, e.g., $[8,9]$ ).

It is also very well known that if $C$ is a nonempty closed convex subset of a Hilbert space $H$ and $P_{C}: H \rightarrow C$ is the metric projection of $H$ onto $C$, then $P_{C}$ is nonexpansive. This fact actually characterizes Hilbert spaces and consequently, it is not available in more general Banach spaces. In this connection, Alber [10] recently introduced a generalized projection operator $\Pi_{C}$ in a Banach space $X$ which is an analogue of the metric projection in Hilbert spaces.

Next, we assume that $X$ is a real reflexive, strictly convex, and smooth Banach space. Let us consider the functional defined as in $[4,5]$ by

$$
\phi(x, y)=\|x\|^{2}-2\langle x, J y\rangle+\|y\|^{2} \text { for } x, y \in E .
$$

Observe that, in a Hilbert space $H,(2.2)$ reduces to $\phi(x, y)=\|x-y\|^{2}, x, y \in H$.

The generalized projection $\Pi_{C}: X \rightarrow C$ is a map that assigns to an arbitrary point $x \in X$ the unique minimum point of the functional $\phi(\cdot, x)$; that is, $\Pi_{C} x=\bar{x}$, where $\bar{x}$ is the unique solution to the minimization problem

$$
\phi(\bar{x}, x)=\min _{y \in C} \phi(y, x)
$$

Remark 2.1. The existence and uniqueness of the element $\bar{x} \in C$ follow from the reflexivity of $X$, the properties of the functional $\phi(\cdot, x)$, and strict monotonicity of the mapping $J$ (see, e.g., [8-12]). In Hilbert spaces, $\Pi_{C}=P_{C}$. It is obvious from the definition of function $\phi$ that

$$
(\|y\|-\|x\|)^{2} \leq \phi(y, x) \leq(\|y\|+\|x\|)^{2} \quad \forall x, y \in X .
$$

Remark 2.2. If $X$ is a reflexive, strictly convex, and smooth Banach space, then for $x, y \in X$, $\phi(x, y)=0$ if and only if $x=y$. It is sufficient to show that if $\phi(x, y)=0$ then $x=y$. From (2.4), we have $\|x\|=\|y\|$. This in turn implies that $\langle x, J y\rangle=\|x\|^{2}=\|J y\|^{2}$. From the smoothness of $X$, we know that $J$ is single valued, and hence we have $J x=J y$. Since $X$ is strictly convex, $J$ is strictly monotone, in particular, $J$ is one to one, which implies that $x=y$; one may consult $[8,9]$ for the details.

Let $C$ be a closed convex subset of $X$, and $T$ a mapping from $C$ into itself. A point $p$ in $C$ is said to be asymptotic fixed point of $T$ [13] if $C$ contains a sequence $\left\{x_{n}\right\}$ which converges weakly to $p$ such that $\lim _{n \rightarrow \infty}\left\|x_{n}-T x_{n}\right\|=0$. The set of asymptotic fixed point of $T$ will be denoted by $\widetilde{F(T)}$. A mapping $T$ from $C$ into itself is said to be relatively nonexpansive $[5,14-$ 16] if $\widetilde{F(T)}=F(T)$ and $\phi(p, T x) \leq \phi(p, x)$ for all $x \in C$ and $p \in F(T)$. The asymptotic behavior of a relatively nonexpansive mapping was studied in [14-16]. 
$T$ is said to be quasi- $\phi$-nonexpansive if $F(T) \neq \emptyset$ and $\phi(p, T x) \leq \phi(p, x)$ for all $x \in C$ and $p \in F(T)$.

Remark 2.3. The class of quasi- $\phi$-nonexpansive mappings is more general than the class of relatively nonexpansive mappings $[5,14-16]$ which requires the strong restriction: $\widetilde{F(T)}=$ $F(T)$.

We present two examples which are closed and quasi- $\phi$-nonexpansive.

Example 2.4. Let $\Pi_{C}$ be the generalized projection from a smooth, strictly convex, and reflexive Banach space $X$ onto a nonempty closed convex subset $C$ of $X$. Then, $\Pi_{C}$ is a closed and quasi- $\phi$-nonexpansive mapping from $X$ onto $C$ with $F\left(\Pi_{C}\right)=C$.

Example 2.5. Let $X$ be a reflexive, strictly convex, and smooth Banach space, and $A \subset X \times X^{*}$ is a maximal monotone mapping such that its zero set $A^{-1} 0$ is nonempty. Then, $J_{r}=(J+r A)^{-1} J$ is a closed and quasi- $\phi$-nonexpansive mapping from $X$ onto $D(A)$ and $F\left(J_{r}\right)=A^{-1} 0$.

Recall that a Banach space $X$ has the property $(\mathrm{K})$ if for any sequence $\left\{x_{n}\right\} \subset X$ and $x \in X$, if $x_{n} \rightarrow x$ weakly and $\left\|x_{n}\right\| \rightarrow\|x\|$, then $\left\|x_{n}-x\right\| \rightarrow 0$. For more information concerning property $(\mathrm{K})$ the reader is referred to [17] and references cited therein.

In order to prove our main result of this paper, we need to the following facts.

Lemma 2.6 (see, e.g., [10-12]). Let $C$ be a convex subset of a real smooth Banach space $X, x \in X$, and $x_{0} \in C$. Then,

$$
\phi\left(x_{0}, x\right)=\inf \{\phi(z, x): z \in C\}
$$

if and only if

$$
\left\langle z-x_{0}, J x_{0}-J x\right\rangle \geq 0, \quad \forall z \in C
$$

Lemma 2.7 (see, e.g., [10-12]). Let $C$ be a convex subset of a real reflexive, strictly convex, and smooth Banach space X. Then the following inequality holds:

$$
\phi\left(y, \Pi_{C} x\right)+\phi\left(\Pi_{C} x, x\right) \leq \phi(y, x)
$$

for all $x \in X$ and $y \in C$.

Now we are in a proposition to prove the main results of this paper.

\section{Main Results}

Theorem 3.1. Let $X$ be a reflexive, strictly convex, smooth Banach space such that $X$ and $X^{*}$ have the property (K). Assume that $C$ is a nonempty closed convex subset of $X$. Let $\left\{T_{i}\right\}_{i=1}^{\infty}: C \rightarrow C$ be an infinitely countable family of closed and quasi- $\phi$-nonexpansive mappings such that $F=\bigcap_{i=1}^{\infty} F\left(T_{i}\right) \neq \emptyset$. 
Assume that $\left\{\alpha_{n, i}\right\}$ are real sequences in $[0,1]$ such that $b_{0, i}=\lim _{\inf _{n \rightarrow \infty}} \alpha_{n, i}<1$. Define a sequence $\left\{x_{n}\right\}$ in $C$ by the following algorithm:

$$
\begin{aligned}
& x_{0} \in X \text { chosen arbitrarily, } \\
& C_{1, i}=C, \quad C_{1}=\bigcap_{i=1}^{\infty} C_{1, i}, \quad x_{1}=\Pi_{C_{1}}\left(x_{0}\right), \\
& y_{n, i}=J^{-1}\left(\alpha_{n, i} J x_{n}+\left(1-\alpha_{n, i}\right) J\left(T_{i} x_{n}\right)\right), \quad n \geq 1, \\
& C_{n+1, i}=\left\{z \in C_{n, i}: \phi\left(z, y_{n, i}\right) \leq \phi\left(z, x_{n}\right)\right\}, \\
& C_{n+1}=\bigcap_{i=1}^{\infty} C_{n+1, i}, \\
& x_{n+1}=\prod_{C_{n+1}} x_{0}, \quad n \geq 0 .
\end{aligned}
$$

Then $\left\{x_{n}\right\}$ converges strongly to $p_{0}=\Pi_{F} x_{0}$, where $\Pi_{F}$ is the generalized projection from $C$ onto $F$.

Proof. We split the proof into six steps.

Step 1. Show that $\Pi_{F} x_{0}$ is well defined for every $x_{0} \in X$.

To this end, we prove first that $F\left(T_{i}\right)$ is closed and convex for any $i \in N$. Let $\left\{p_{n}\right\}$ be a sequence in $F\left(T_{i}\right)$ with $p_{n} \rightarrow p$ as $n \rightarrow \infty$, we prove that $p \in F\left(T_{i}\right)$. From the definition of quasi- $\phi$-nonexpansive mappings, one has $\phi\left(p_{n}, T_{i} p\right) \leq \phi\left(p_{n}, p\right)$, which implies that $\phi\left(p_{n}, T_{i} p\right) \rightarrow 0$ as $n \rightarrow \infty$. Noticing that

$$
\phi\left(p_{n}, T_{i} p\right)=\left\|p_{n}\right\|^{2}-2\left\langle p_{n}, J\left(T_{i} p\right)\right\rangle+\left\|T_{i} p\right\|^{2} .
$$

By taking limit in (3.2), we have

$$
\lim _{n \rightarrow \infty} \phi\left(p_{n}, T_{i} p\right)=\|p\|^{2}-2\left\langle p, J\left(T_{i} p\right)\right\rangle+\left\|T_{i} p\right\|^{2}=\phi\left(p, T_{i} p\right)
$$

Hence $\phi\left(p, T_{i} p\right)=0$. It implies that $p=T_{i} p$ for all $i \in N$. We next show that $F\left(T_{i}\right)$ is convex. To this end, for arbitrary $p_{1}, p_{2} \in F\left(T_{i}\right), t \in(0,1)$, putting $p_{3}=t p_{1}+(1-t) p_{2}$, we prove that $T_{i} p_{3}=p_{3}$. Indeed, by using the definition of $\phi(x, y)$, we have

$$
\begin{aligned}
\phi\left(p_{3}, T_{i} p_{3}\right) & =\left\|p_{3}\right\|^{2}-2\left\langle p_{3}, J\left(T_{i} p_{3}\right)\right\rangle+\left\|T_{i} p_{3}\right\|^{2} \\
& =\left\|p_{3}\right\|^{2}-2\left\langle t p_{1}+(1-t) p_{2}, J\left(T_{i} p_{3}\right)\right\rangle+\left\|T_{i} p_{3}\right\|^{2} \\
& =\left\|p_{3}\right\|^{2}-2 t\left\langle p_{1}, J\left(T_{i} p_{3}\right)\right\rangle-2(1-t)\left\langle p_{2}, J\left(T_{i} p_{3}\right)\right\rangle+\left\|T_{i} p_{3}\right\|^{2} \\
& =\left\|p_{3}\right\|^{2}+t \phi\left(p_{1}, T_{i} p_{3}\right)+(1-t) \phi\left(p_{2}, T_{i} p_{3}\right)-t\left\|p_{1}\right\|^{2}-(1-t)\left\|p_{2}\right\|^{2} \\
& \leq\left\|p_{3}\right\|^{2}+t \phi\left(p_{1}, p_{3}\right)+(1-t) \phi\left(p_{2}, p_{3}\right)-t\left\|p_{1}\right\|^{2}-(1-t)\left\|p_{2}\right\|^{2} \\
& =\left\|p_{3}\right\|^{2}-2\left\langle p_{3}, J p_{3}\right\rangle+\left\|p_{3}\right\|^{2} \\
& =0 .
\end{aligned}
$$


This implies that $T_{i} p_{3}=p_{3}$. Hence $F\left(T_{i}\right)$ is closed and convex for all $i \in N$ and consequently $F=\bigcap_{i=1}^{\infty} F\left(T_{i}\right)$ is closed and convex. By our assumption that $F=\bigcap_{i=1}^{\infty} F\left(T_{i}\right) \neq \emptyset$, we have $\Pi_{F} x_{0}$ is well defined for every $x_{0} \in X$.

Step 2. Show that $C_{n}$ is closed and convex for each $n \geq 1$.

It suffices to show that for any $i \in N, C_{n, i}$ is closed and convex for every $n \geq 1$. This can be proved by induction on $n$. In fact, for $n=1, C_{1, i}=C$ is closed and convex. Assume that $C_{n, i}$ is closed and convex for some $n \geq 1$. For $z \in C_{n+1, i}$, one obtains that

$$
\phi\left(z, y_{n, i}\right) \leq \phi\left(z, x_{n}\right)
$$

is equivalent to

$$
2\left\langle z, J x_{n}-J y_{n, i}\right\rangle \leq\left\|x_{n}\right\|^{2}-\left\|y_{n, i}\right\|^{2}
$$

It is easy to see that $C_{n+1, i}$ is closed and convex. Then, for all $n \geq 1, C_{n, i}$ is closed and convex. Consequently, $C_{n}=\bigcap_{i=1}^{\infty} C_{n, i}$ is closed and convex for all $n \geq 1$.

Step 3. Show that $F=\bigcap_{i=1}^{\infty} F\left(T_{i}\right) \subset \bigcap_{n=1}^{\infty} C_{n}=D$.

It suffices to show that for any $i \in N, F \subset C_{n, i}$ for every $n \geq 1$. For any $c_{0} \in F$, from the definition of quasi- $\phi$-nonexpansive mappings, we have $\phi\left(c_{0}, T_{i} x\right) \leq \phi\left(c_{0}, x\right)$, for all $x \in C$ and $i \in N$. Noting that for any $x \in C$ and $\alpha \in[0,1]$, we have

$$
\begin{aligned}
\phi\left(c_{0},\right. & \left.J^{-1}\left(\alpha J x+(1-\alpha) J\left(T_{i} x\right)\right)\right) \\
& =\left\|c_{0}\right\|^{2}-2\left\langle c_{0}, \alpha J x+(1-\alpha) J\left(T_{i} x\right)\right\rangle+\left\|J^{-1}\left(\alpha J x+(1-\alpha) J\left(T_{i} x\right)\right)\right\|^{2} \\
& \leq\left\|c_{0}\right\|^{2}-2\left\langle c_{0}, \alpha J x+(1-\alpha) J\left(T_{i} x\right)\right\rangle+\alpha\|x\|^{2}+(1-\alpha)\left\|T_{i} x\right\|^{2} \\
& =\alpha \phi\left(c_{0}, x\right)+(1-\alpha) \phi\left(c_{0}, T_{i} x\right) \\
& \leq \alpha \phi\left(c_{0}, x\right)+(1-\alpha) \phi\left(c_{0}, x\right) \\
& =\phi\left(c_{0}, x\right)
\end{aligned}
$$

which implies that $c_{0} \in C_{n, i}$ and consequently $F \subset C_{n, i}$. So $F \subset \bigcap_{n=1}^{\infty} C_{n}$. Hence $x_{n+1}=\prod_{C_{n+1}} x_{0}$ is well defined for each $n \geq 0$. Therefore, the iterative algorithm (3.1) is well defined.

Step 4. Show that $\left\|x_{n}-p_{0}\right\| \rightarrow 0$, where $p_{0}=\Pi_{D} x_{0}$.

From Steps 2 and 3, we obtain that $D$ is a nonempty, closed, and convex subset of $C$. Hence $\Pi_{D} x_{0}$ is well defined for every $x_{0} \in C$. From the construction of $C_{n}$, we know that

$$
C \supset C_{1} \supset C_{2} \supset \cdots
$$


Let $p_{0}=\Pi_{D} x_{0}$, where $p_{0} \in C$ is the unique element that satisfies $\inf _{x \in D} \phi\left(x, x_{0}\right)=\phi\left(p_{0}, x_{0}\right)$. Since $x_{n}=\Pi_{C_{n}} x_{0}$, by Lemma 2.7, we have

$$
\phi\left(x_{n}, x_{0}\right) \leq \phi\left(x_{n+1}, x_{0}\right) \leq \cdots \leq \phi\left(p_{0}, x_{0}\right)
$$

By the reflexivity of $X$, we can assume that $x_{n} \rightarrow g_{1} \in X$ weakly. Since $C_{j} \subset C_{n}$, for $j \geq n$, we have $x_{j} \in C_{n}$ for $j \geq n$. Since $C_{n}$ is closed and convex, by the Marzur theorem, $g_{1} \in C_{n}$ for any $n \in N$. Hence $g_{1} \in D$. Moreover, by using the weakly lower semicontinuity of the norm on $X$ and (3.9), we obtain

$$
\begin{aligned}
\phi\left(p_{0}, x_{0}\right) & \leq \phi\left(g_{1}, x_{0}\right) \leq \liminf _{n \rightarrow \infty} \phi\left(x_{n}, x_{0}\right) \\
& \leq \limsup _{n \rightarrow \infty} \phi\left(x_{n}, x_{0}\right) \leq \inf _{x \in D} \phi\left(x, x_{0}\right)=\phi\left(p_{0}, x_{0}\right),
\end{aligned}
$$

which implies that $\lim _{n \rightarrow \infty} \phi\left(x_{n}, x_{0}\right)=\phi\left(p_{0}, x_{0}\right)=\phi\left(g_{1}, x_{0}\right)=\inf _{x \in D} \phi\left(x, x_{0}\right)$. By using Lemma 2.6, we have

$$
\left\langle p_{0}-g_{1}, J p_{0}-J g_{1}\right\rangle=0,
$$

and hence $p_{0}=g_{1}$, since $J$ is strictly monotone.

Further, by the definition of $\phi$, we have

$$
\lim _{n \rightarrow \infty}\left(\left\|x_{n}\right\|^{2}-2\left\langle x_{n}, J x_{0}\right\rangle+\left\|x_{0}\right\|^{2}\right)=\left(\left\|p_{0}\right\|^{2}-2\left\langle p_{0}, J x_{0}\right\rangle+\left\|x_{0}\right\|^{2}\right)
$$

which shows that $\lim _{n \rightarrow \infty}\left\|x_{n}\right\|=\left\|p_{0}\right\|$. By the property $(\mathrm{K})$ of $X$, we have $\left\|x_{n}-p_{0}\right\| \rightarrow 0$, where $p_{0}=\Pi_{D} x_{0}$.

Step 5. Show that $p_{0}=T_{i} p_{0}$, for any $i \in N$.

Since $x_{n+1} \in C_{n+1}=\bigcap_{i=1}^{\infty} C_{n+1, i}$ for all $n \geq 0$ and $i \in N$, we have

$$
0 \leq \phi\left(x_{n+1}, y_{n, i}\right) \leq \phi\left(x_{n+1}, x_{n}\right) .
$$

Since $\left\|x_{n}-p_{0}\right\| \rightarrow 0, \phi\left(x_{n+1}, x_{n}\right) \rightarrow 0$ and consequently

$$
\phi\left(x_{n+1}, y_{n, i}\right) \longrightarrow 0
$$

Note that $0 \leq\left(\left\|x_{n+1}\right\|-\left\|y_{n, i}\right\|\right)^{2} \leq \phi\left(x_{n+1}, y_{n, i}\right)$. Hence $\left\|y_{n, i}\right\| \rightarrow\left\|p_{0}\right\|$ and consequently $\left\|J\left(y_{n, i}\right)\right\| \rightarrow\left\|J p_{0}\right\|$. This implies that $\left\{J\left(y_{n, i}\right)\right\}$ is bounded. Since $X$ is reflexive, $X^{*}$ is also reflexive. So we can assume that

$$
J\left(y_{n, i}\right) \longrightarrow f_{0} \in X^{*}
$$


weakly. On the other hand, in view of the reflexivity of $X$, one has $J(X)=X^{*}$, which means that for $f_{0} \in X^{*}$, there exists $x \in X$, such that $J(x)=f_{0}$. It follows that

$$
\begin{aligned}
\lim _{n \rightarrow \infty} \phi\left(x_{n+1}, y_{n, i}\right) & =\lim _{n \rightarrow \infty}\left[\left\|x_{n+1}\right\|^{2}-2\left\langle x_{n+1}, J\left(y_{n, i}\right)\right\rangle+\left\|y_{n, i}\right\|^{2}\right] \\
& =\lim _{n \rightarrow \infty}\left[\left\|x_{n+1}\right\|^{2}-2\left\langle x_{n+1}, J\left(y_{n, i}\right)\right\rangle+\left\|J\left(y_{n, i}\right)\right\|^{2}\right] \\
& \geq\left\|p_{0}\right\|^{2}-2\left\langle p_{0}, f_{0}\right\rangle+\left\|f_{0}\right\|^{2} \\
& =\left\|p_{0}\right\|^{2}-2\left\langle p_{0}, J x\right\rangle+\|J x\|^{2} \\
& =\phi\left(p_{0}, x\right),
\end{aligned}
$$

where we used the weakly lower semicontinuity of the norm on $X^{*}$. From (3.14), we have $\phi\left(p_{0}, x\right)=0$ and consequently $p_{0}=x$, which implies that $f_{0}=J p_{0}$. Hence

$$
J\left(y_{n, i}\right) \longrightarrow J p_{0} \in X^{*}
$$

weakly. Since $\left\|J\left(y_{n, i}\right)\right\| \rightarrow\left\|J p_{0}\right\|$ and $X^{*}$ has the property $(\mathrm{K})$, we have

$$
\left\|J\left(y_{n, i}\right)-J p_{0}\right\| \longrightarrow 0
$$

Since $\left\|x_{n}-p_{0}\right\| \rightarrow 0$, noting that $J: X \rightarrow X^{*}$ is demi-continuous, we have

$$
J x_{n} \longrightarrow J p_{0} \in X^{*}
$$

weakly. Noticing that

$$
\left|\left\|J x_{n}\right\|-\left\|J p_{0}\right\|\right|=\left|\left\|x_{n}\right\|-\left\|p_{0}\right\|\right| \leq\left\|x_{n}-p_{0}\right\| \longrightarrow 0,
$$

which implies that $\left\|J x_{n}\right\| \rightarrow\left\|J p_{0}\right\|$. By using the property $(\mathrm{K})$ of $X^{*}$, we have

$$
\left\|J x_{n}-J p_{0}\right\| \longrightarrow 0 \text {. }
$$

From (3.1), (3.18), (3.21), and $b_{0, i}=\liminf _{n \rightarrow \infty} \alpha_{n, i}<1$, we have

$$
\left\|J\left(T_{i} x_{n}\right)-J p_{0}\right\| \longrightarrow 0
$$

Since $J^{-1}: X^{*} \rightarrow X$ is demi-continuous, we have

$$
T_{i} x_{n} \longrightarrow p_{0}
$$

weakly in X. Moreover,

$$
\left|\left\|T_{i} x_{n}\right\|-\left\|p_{0}\right\|\right|=\left|\left\|J\left(T_{i} x_{n}\right)\right\|-\left\|J p_{0}\right\|\right| \leq\left\|J\left(T_{i} x_{n}\right)-J p_{0}\right\| \longrightarrow 0,
$$


which implies that $\left\|T_{i} x_{n}\right\| \rightarrow\left\|p_{0}\right\|$. By the property (K) of $X$, we have

$$
T_{i} x_{n} \longrightarrow p_{0}
$$

From $\left\|x_{n}-p_{0}\right\| \rightarrow 0$ and the closeness property of $T_{i}$, we have

$$
T_{i} p_{0}=p_{0},
$$

which implies that $p_{0} \in F=\bigcap_{i=1}^{\infty} F\left(T_{i}\right)$.

Step 6. Show that $p_{0}=\Pi_{F} x_{0}$.

It follows from Steps 3, 4, and 5 that

$$
\phi\left(p_{0}, x_{0}\right) \leq \phi\left(\Pi_{F} x_{0}, x_{0}\right) \leq \phi\left(p_{0}, x_{0}\right)
$$

which implies that $\phi\left(\Pi_{F} x_{0}, x_{0}\right)=\phi\left(p_{0}, x_{0}\right)$. Hence, $p_{0}=\Pi_{F} x_{0}$. Then $\left\{x_{n}\right\}$ converges strongly to $p_{0}=\Pi_{F} x_{0}$. This completes the proof.

From Theorem 3.1, we can obtain the following corollary.

Corollary 3.2. Let $X$ be a reflexive, strictly convex and smooth Banach space such that both $X$ and $X^{*}$ have the property (K). Assume that $C$ is a nonempty closed convex subset of $X$. Let $T: C \rightarrow C$ be a closed and quasi- $\phi$-nonexpansive mapping. Assume that $\left\{\alpha_{n}\right\}$ is a sequence in $[0,1]$ such that $b_{0}=\liminf _{n \rightarrow \infty} \alpha_{n}<1$. Define a sequence $\left\{x_{n}\right\}$ in $C$ by the following algorithm:

$$
\begin{aligned}
& x_{0} \in C \text { chosen arbitrarily, } \\
& C_{1}=C, \quad x_{1}=\Pi_{C_{1}}\left(x_{0}\right), \\
& y_{n}=J^{-1}\left(\alpha_{n} J x_{n}+\left(1-\alpha_{n}\right) J\left(T x_{n}\right)\right), \quad n \geq 1, \\
& C_{n+1}=\left\{z \in C_{n}: \phi\left(z, y_{n}\right) \leq \phi\left(z, x_{n}\right)\right\}, \\
& x_{n+1}=\prod_{C_{n+1}} x_{0}, \quad n \geq 0 .
\end{aligned}
$$

Then $\left\{x_{n}\right\}$ converges strongly to $p_{0}=\Pi_{F(T)} x_{0}$, where $\Pi_{F(T)}$ is the generalized projection from $C$ onto $F(T)$.

Remark 3.3. Theorem 3.1 and its corollary improve and extend Theorems MT and QS at several aspects.

(i) From uniformly convex and uniformly smooth Banach spaces extend to reflexive, strictly convex and smooth Banach spaces with the property (K). In Theorem 3.1 and its corollary the hypotheses on $X$ are weaker than the usual assumptions of uniform convexity and uniform smoothness. For example, any strictly convex, reflexive and smooth Musielak-Orlicz space satisfies our assumptions [17] while, in general, these spaces need not to be uniformly convex or uniformly smooth. 
(ii) From relatively nonexpansive mappings extend to closed and quasi- $\phi$-nonexpansive mappings.

(iii) The continuity assumption on mapping $T$ in Theorem QS is removed.

(iv) Relax the restriction on $\left\{\alpha_{n}\right\}$ from $\lim \sup _{n \rightarrow \infty} \alpha_{n}<1$ to $\liminf _{n \rightarrow \infty} \alpha_{n}<1$.

Remark 3.4. Corollary 3.2 presents some affirmative answers to Questions 1 and 2.

\section{Applications}

In this section, we present some applications of the main results in Section 3.

Theorem 4.1. Let $X$ be a reflexive, strict, and smooth Banach space that both $X$ and $X^{*}$ have the property $(K)$, and let $C$ be a nonempty closed convex subset of $X$. Let $\left\{f_{i}\right\}_{i \in \mathbb{N}}: X \rightarrow(-\infty,+\infty]$ be a family of proper, lower semicontinuous, and convex functionals. Assume that the common fixed point set $F=\bigcap_{i \in \mathbb{N}} F\left(J_{i}\right)$ is nonempty, where $J_{i}=\left(J+r_{i} \partial f_{i}\right)^{-1} J$ for $r_{i}>0$ and $i \in \mathbb{N}$. Let $\left\{x_{n}\right\}$ be a sequence generated by the following manner:

$$
\begin{aligned}
& x_{0} \in X \text { chosen arbitrarily, } \\
& C_{1, i}=C, \quad C_{1}=\bigcap_{i=1}^{\infty} C_{1, i}, \quad x_{1}=\prod_{C_{1}}\left(x_{0}\right), \\
& y_{n, i}=J^{-1}\left(\alpha_{n, i} J x_{n}+\left(1-\alpha_{n, i}\right) J z_{n, i}\right), \quad n \geq 1, \\
& z_{n, i}=\underset{z \in X}{\operatorname{argmin}}\left\{f_{i}(z)+\frac{1}{2 r_{i}}\|z\|^{2}-\frac{1}{r_{i}}\left\langle z, J x_{n}\right\rangle\right\}, \\
& C_{n+1, i}=\left\{z \in C_{n, i}: \phi\left(z, y_{n, i}\right) \leq \phi\left(z, x_{n}\right)\right\}, \\
& C_{n+1}=\bigcap_{i=1}^{\infty} C_{n+1, i}, \\
& x_{n+1}=\prod_{C_{n+1}} x_{0}, \quad n \geq 0,
\end{aligned}
$$

where $\left\{\alpha_{n, i}\right\}$ satisfies the restriction: $0 \leq \alpha_{n, i}<1$ and $\liminf _{n \rightarrow \infty} \alpha_{n, i}<1$. Then $\left\{x_{n}\right\}$ defined by (4.1) converges strongly to a minimizer $\Pi_{F} x_{0}$ of the family $\left\{f_{i}\right\}_{i \in \mathbb{N}}$.

Proof. By a result of Rockafellar [18], we see that $\partial f_{i}: X \rightarrow 2^{X^{*}}$ is a maximal monotone mapping for every $i \in \mathbb{N}$. It follows from Example 2.5 that $J_{i}: X \rightarrow X$ is a closed and quasi$\phi$-nonexpansive mapping for every $i \in \mathbb{N}$. Notice that

$$
z_{n, i}=\underset{z \in X}{\operatorname{argmin}}\left\{f_{i}(z)+\frac{1}{2 r_{i}}\|z\|^{2}-\frac{1}{r_{i}}\left\langle z, J x_{n}\right\rangle\right\}
$$


is equivalent to

$$
0 \in \partial f_{i}\left(z_{n, i}\right)+\frac{1}{r_{i}} J z_{n, i}-\frac{1}{r_{i}} J x_{n}, \quad n, i \in \mathbb{N}
$$

and the last inclusion relation is equivalent to

$$
z_{n, i}=\left(J+r_{i} \partial f_{i}\right)^{-1} J x_{n}=J_{i} x_{n}
$$

Now the desired conclusion follows from Theorem 3.1. This completes the proof.

\section{Acknowledgment}

This work was supported by the National Natural Science Foundation of China under Grant (10771050).

\section{References}

[1] Y. Haugazeau, Sur les inéquations variationnelles et la minimisation de fonctionnelles convexes, Thése, Université de Paris, Paris, France.

[2] X. L. Qin and Y. F. Su, "Strong convergence theorems for relatively nonexpansive mappings in a Banach space," Nonlinear Analysis, vol. 67, no. 6, pp. 1958-1965, 2007.

[3] K. Nakajo and W. Takahashi, "Strong convergence theorems for nonexpansive mappings and nonexpansive semigroups," Journal of Mathematical Analysis and Applications, vol. 279, no. 2, pp. 372 379, 2003.

[4] M. Y. Carlos and H. K. Xu, "Strong convergence of the CQ method for fixed point iteration processes," Nonlinear Analysis, vol. 64, no. 11, pp. 2400-2411, 2006.

[5] S. Matsushita and W. Takahashi, "A strong convergence theorem for relatively nonexpansive mappings in a Banach space," Journal of Approximation Theory, vol. 134, no. 2, pp. 257-266, 2005.

[6] Y. F. Su and X. L. Qin, "Strong convergence of modified Ishikawa iterations for nonlinear mappings," Proceedings of the Indian Academy of Sciences, Mathematical Sciences, vol. 117, no. 1, pp. 97-107, 2007.

[7] Y. F. Su, D. X. Wang, and M. J. Shang, "Strong convergence of monotone hybrid algorithm for hemirelatively nonexpansive mappings," Fixed Point Theory and Applications, vol. 2008, Article ID 284613, 8 pages, 2008.

[8] I. Cioranescu, Geometry of Banach Spaces, Duality Mappings and Nonlinear Problems, vol. 62, Kluwer Academic Publishers Group, Dordrecht, The Netherlands, 1990.

[9] W. Takahashi, Nonlinear Functional Analysis, Yokohama Publishers, Yokohama, Japan, 2000.

[10] Ya. I. Alber, "Metric and generalized projection operators in Banach spaces: properties and applications," in Theory and Applications of Nonlinear Operators of Accretive and Monotone Type, A. G. Kartsatos, Ed., vol. 178, pp. 15-50, Marcel Dekker, New York, NY, USA, 1996.

[11] Ya. I. Alber and S. Reich, "An iterative method for solving a class of nonlinear operator equations in Banach spaces," Panamerican Mathematical Journal, vol. 4, no. 2, pp. 39-54, 1994.

[12] S. Kamimura and W. Takahashi, "Strong convergence of a proximal-type algorithm in a Banach space," SIAM Journal on Optimization, vol. 13, no. 3, pp. 938-945, 2002.

[13] S. Reich, "A weak convergence theorem for the alternating method with Bregman distances," in Theory and Applications of Nonlinear Operators of Accretive and Monotone Type, A. G. Kartsatos, Ed., vol. 178 of Lecture Notes in Pure and Applied Mathmatics, pp. 313-318, Marcel Dekker, New York, NY, USA, 1996.

[14] D. Butnariu, S. Reich, and A. J. Zaslavski, "Asymptotic behavior of relatively nonexpansive operators in Banach spaces," Journal of Applied Analysis, vol. 7, no. 2, pp. 151-174, 2001.

[15] D. Butnariu, S. Reich, and A. J. Zaslavski, "Weak convergence of orbits of nonlinear operators in reflexive Banach spaces," Numerical Functional Analysis and Optimization, vol. 24, no. 5-6, pp. 489-508, 2003. 
[16] Y. Censor and S. Reich, "Iterations of paracontractions and firmly nonexpansive operators with applications to feasibility and optimization," Optimization, vol. 37, no. 4, pp. 323-339, 1996.

[17] H. Hudzik, W. Kowalewski, and G. Lewicki, "Approximate compactness and full rotundity in Musielak-Orlicz spaces and Lorentz-Orlicz spaces," Zeitschrift fuer Analysis und ihre Anwendungen, vol. 25, no. 2, pp. 163-192, 2006.

[18] R. T. Rockafellar, "On the maximal monotonicity of subdifferential mappings," Pacific Journal of Mathematics, vol. 33, pp. 209-216, 1970. 